\title{
LAS INTERACCIONES DE LOS ESTUDIANTES EN ENTORNOS VIRTUALES DE APRENDIZAJE: HERRAMIENTAS INFORMÁTICAS PARA SU SEGUIMIENTO Y DIRECCIÓN
}

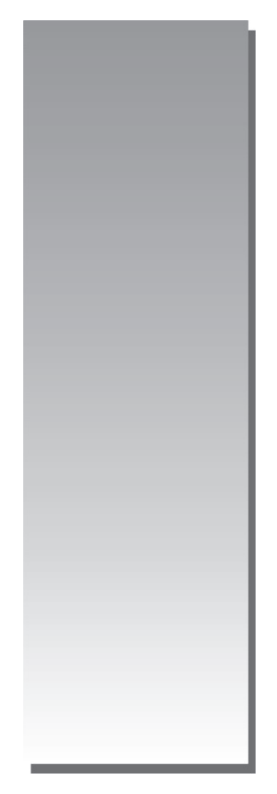

Juan Castillo Maza (*)

E-mail: jcastillom@unmsm.edu.pe

Vivian Estrada Sentí (**)

E-mail:vestrada@reduniv.edu.cu

Walfredo González Hernández (****)

E-mail:walfredo.glez@umcc.cu

Rolando Rodríguez Ortega (******)

E-mail: rolando.rodriguez@umcc.cu

Lázaro Tió Torriente $\left(^{(* * * * * * * * *)}\right.$

E-mail: ltio@umcc.cu

\begin{abstract}
RESUMEN
Contar con herramientas para guiar, controlar y evaluar las interacciones de los estudiantes en foros de entonos virtuales de aprendizaje (EVA) resulta indispensable para contribuir a un verdadero proceso de aprendizaje. Este trabajo tiene como objetivo brindar un instrumento y una herramienta para realizar el análisis del contenido de las interacciones y la actuación de los estudiantes: el instrumento es el foro del entorno virtual de aprendizaje (denominado los diez emoticones) y la herramienta informática proporciona una representación gráfica de diagramas estructurales, tanto del modelo de interacción del estudiante como del grupo que desarrolla actividades en el foro del EVA. La aplicación del instrumento y de la herramienta informática facilitan al profesor supervisar, guiar y evaluar el proceso de aprendizaje, formación y desarrollo del grupo en el EVA.
\end{abstract}

Palabras clave: Entorno virtual de aprendizaje, grupo, formación, desarrollo.

(*) Doctor en Ciencias Contables y Empresariales. Profesor Principal de la Universidad Nacional Mayor de San Marcos.

$(* *)$ Doctora en Ciencias Técnicas. Asesora de Tecnología Educativa del Ministerio de Educación Superior de Cuba.

$\left({ }^{(* * *)}\right)$ Doctor en Ciencias Pedagógicas. Profesor de Informática de la Universidad de Matanzas (Cuba).

(****) Ingeniero en Informática. Profesor de Informática de la Universidad de Matanzas (Cuba).

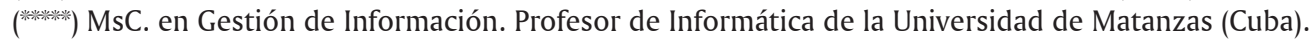




\begin{abstract}
Have tools to guide, monitor and assess the interactions of students in virtual forums to sing learning is essential to support real learning process. This paper aims to provide two tools with which manages to make the content analysis of the interactions and student performance in the forum of the virtual learning environment (called the ten emoticons), and another that provides a graphical representation of structural diagrams, both the model of student interaction and group that is active in the forum of the virtual learning environment (VLE). The application of the instrument and the computer tool helps the teacher supervise, guide and evaluate the process of learning and training and development in the EVA group.
\end{abstract}

Keywords: Virtual environment for learning, group, formation, development.

\section{INTRODUCCIÓN}

Los aspectos didácticos correspondientes al proceso de conducción del trabajo grupal en los centros de educación superior adquieren cada día mayor importancia en el proceso docente educativo. En la práctica educativa predominan los métodos tradicionales en detrimento de las vías que propician la interacción, el intercambio de conocimientos, la formación y desarrollo del grupo.

El aprendizaje en grupo (en ambientes colaborativos) busca propiciar espacios que contribuyan al desarrollo de habilidades individuales a partir de la discusión entre estudiantes al momento de explorar nuevos conceptos, siendo cada quien responsable de su propio aprendizaje. Se pretende que estos ambientes sean ricos en posibilidades y, más que organizadores de la información, propicien el crecimiento del grupo.

Diferentes teorías del aprendizaje encuentran aplicación en los ambientes de trabajo en grupo; entre éstas tenemos los enfoques de Piaget y Vigotsky basados en la interacción social. El aprendizaje es un proceso individual que puede ser enriquecido con actividades colaborativas tendientes a desarrollar habilidades personales y de trabajo en grupo en el individuo. El desarrollo grupal no es espontáneo y la acumulación de experiencias e investigaciones al respecto permiten establecer elementos de juicio para incidir sobre su formación y desarrollo, para hacerlo más productivo desde el punto de vista educativo y respecto con su contribución al aprendizaje.

En el ámbito docente surgen nuevas aplicaciones que contribuyen al trabajo en grupo de alumnos y profesores, tanto en el entorno del aula como a distancia, manteniendo la estructura tradicional del grupo docente previamente establecida o en grupos libres formados por la elección individual de sus miembros. Estas aplicaciones constituyen todo un marco de investigación relacionado con el soporte informático, los modelos educativos, la calidad del proceso enseñanza-aprendizaje y su repercusión para la formación y desarrollo del grupo.

En la literatura consultada se encontraron diversas investigaciones desarrolladas sobre los procesos de enseñanza y aprendizaje en EVA y se pueden apreciar diferentes instrumentos elaborados para llevar a cabo el análisis de las interacciones. García, M. C. ${ }^{1}$; Ehuletche, M. A. y otros ${ }^{2}$; Gros, B. y Silva, J. ${ }^{3}$ presentan una recopilación de los principales instrumentos desarrollados a lo largo de las dos últimas décadas para el análisis de la interacción on line. En todos estos estudios las alternativas son variadas, fundamentalmente en relación a las unidades de análisis utilizadas, las que pueden afectar la fiabilidad de los estudios (en unos casos se han escogido unidades temáticas, en otros se analizan mensajes completos). Los autores consideran que es preciso avanzar en la clarificación de las unidades de análisis seleccionadas y su interrelación con los modelos teóricos utilizados. Además, el grado de replicabilidad de la mayoría de los estudios es muy bajo.

Entre las limitaciones o problemas detectados en estas propuestas se pueden señalar el carácter exploratorio asociado a prototipos de investigación; dificultades con la unidad de análisis y el hecho que, por lo general, solo registran los elementos cuantitativos del resultado de las interacciones.

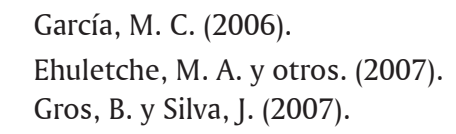


En la literatura consultada se encontraron varias implementaciones de sistemas y herramientas informáticas de análisis de las interacciones colaborativas para apoyar a los profesores y estudiantes en el ámbito de la educación. Entre ellas tenemos los trabajos de Martínez, A. ${ }^{4}$; Domínguez, D. y Alonso, L. ${ }^{5}$; Vélez, O. y otros ${ }^{6}$; Costaguta, R. ${ }^{7}$; Mazza, R. y Dimitrova, V. ${ }^{8}$; Silva, J. ${ }^{9}$; Monguet, J. ${ }^{10}$; Yoo, J., y otros ${ }^{11}$; Lugo, L. ${ }^{12}$

Los autores del trabajo consideran que las propuestas anteriores no están encaminadas a la formación, evolución y desarrollo del grupo y que, en la representación gráfica de los modelos, no se formulan aspectos tales como lo cognitivo, las preferencias, las emociones, la aceptación y la equivalencia que definen el estado de la colaboración en el grupo, elementos importantes que ayudan al profesor a tomar decisiones oportunas dirigidas a formar y desarrollar al grupo en el EVA y guiar su aprendizaje.

Los autores coinciden con Marcelo, C. y Perera, $\mathrm{H} .{ }^{13}$; Paz, V. ${ }^{14}$; y Eric, B. ${ }^{15}$ en que es fundamental que un sistema de trabajo en grupo proporcione herramientas informáticas para favorecer el seguimiento detallado del desarrollo de las actividades de aprendizaje, y así realizar un control y análisis de todo el proceso, la evolución, evaluación y desarrollo del grupo, así como los conocimientos adquiridos, favoreciendo la toma de decisiones. Para ello los autores de este trabajo elaboramos un instrumento denominado los diez emoticones y una herramienta informática llamada Net@nalisis las que se presentarán en este artículo.

\section{MÉTODOS UTILIZADOS}

Se empleó la estrategia de investigación acción para indagar cómo se lleva a cabo el proceso de formación y desarrollo del grupo con estudiantes de la carrera de Ingeniería Informática en el EVA en la Universidad de Matanzas "Camilo Cienfuegos" (UMCC) de Cuba, mediante procedimientos de

4 Martínez, A. (2003).

5 Domínguez, D. y Alonso, L. (2004).

6 Vélez, O. y otros (2004).

7 Costaguta, R., (2006).

8 Mazza, R. y Dimitrova, V. (2007).

9 Silva, J. (2007).

10 Monguet, J. (2008).

11 Yoo, J., y otros. (2009), pp. 3362-3370.

12 Lugo, L (2009).

13 Marcelo, C. y Perera, H. (2006).

14 Paz, V. (2007), pp. 205-219.

15 Eric, B. (2008). acción sistemática, la reflexión sostenida y activa de todos los involucrados en el proceso, que se orienta a estimular la práctica transformadora y el cambio.

Para evaluar la efectividad de la metodología para la formación y desarrollo del grupo en el EVA, se trabajó sobre la plataforma Moodle, y se desarrolló un cuasi-experimento en el curso pasado con los estudiantes de cuarto año de la carrera de Informática. Como población se consideró la matrícula total del grupo, 34 estudiantes, constituyendo la muestra el $100 \%$ de la población.

Los principales métodos y técnicas de investigación aplicados fueron los siguientes:

\section{- Métodos teóricos}

- Análisis - Síntesis: Lo que permitió llegar a conclusiones a partir del estudio realizado del tema objeto de investigación con diferentes fuentes de información.

- Inductivo - Deductivo: Para realizar el tránsito de lo general a lo particular y viceversa al establecer los nexos entre las características del grupo a partir de los indicadores.

- Histórico - Lógico: Para conocer el comportamiento y evolución de las diferentes posiciones respecto al estudio de los grupos.

- Modelación: Para verificar y validar la herramienta informática elaborada y confeccionar la metodología dirigida a la formación y desarrollo del grupo en el EVA.

\section{- Métodos empíricos}

- Observación (directa, abierta, sistemática y participante): Para recoger información y dar seguimiento a los modos de actuación de los estudiantes.

- Encuesta: Para conocer la motivación, los niveles de satisfacción e insatisfacción y las preferencias de los estudiantes en el uso y la realización de las actividades en el EVA.

- Entrevista abierta: Para obtener información a partir de la experiencia de los profesores.

- Análisis de documentos: Para extraer la información necesaria que permita realizar el proceso de intervención pedagógica.

- Triangulación metodológica: Para contrastar los resultados después de aplicar distintos instrumentos. 
- Criterio de expertos: Para enriquecer y evaluar la fiabilidad de las categorías e indicadores utilizados en el instrumento y el valor práctico de la herramienta informática.

- Cuasi-experimento pedagógico: Para evaluar en la práctica la contribución de la metodología al proceso de formación y desarrollo del grupo con estudiantes de la carrera de Ingeniería informática en el EVA en la UMCC.

\section{- Métodos estadísticos}

- Análisis descriptivo: Para determinar la frecuencia y el porcentaje a partir del diagnóstico y la evaluación práctica de la metodología para arribar a conclusiones.

- Método Delphi: Para valorar el instrumento y la herramienta informática elaborados.

\section{- Métodos matemáticos}

- La teoría de grafos y matrices: Para la representación de los modelos de interacción y el análisis e interpretación a través de las medidas del Análisis de Redes Sociales (ARS).

\section{- Técnicas cualitativas o cuantitativas}

- Técnica de Iadov: Para conocer el nivel de satisfacción e insatisfacción de los estudiantes en el uso del EVA sobre las plataformas interactivas disponibles en la UMCC.

- Grupo Focal: Para determinar las categorías e indicadores utilizados en el instrumento.

\section{RESULTADOS Y DISCUSIÓN}

\section{Herramienta los diez emoticones}

Diferentes autores han destacado el uso de métodos de intervención: Fuentes, M.16; Soller, A. y otros ${ }^{17}$; Aguilar, A. R. ${ }^{18}$; Cornejo, M. J. ${ }^{19}$, entre otros. A partir de estos criterios y las consideraciones de los autores del trabajo se elaboró un instrumento de intervención con el objetivo de guiar, controlar, evaluar, potenciar, acelerar y/o corregir el desarrollo grupal, apoyándose en el contenido de las interacciones de estudiantes y profesores en el foro del EVA.

16 Fuentes, M. (2001).

17 Soller, A. y otros (2005), pp. 261-290.

18 Aguilar, A. R. (2005).

19 Cornejo, M. J. (2006).

20 Rodríguez, M. (2008).
El instrumento elaborado utiliza como referentes teóricos las técnicas de la dinámica de grupos: los seis sombreros para pensar y los seis pares de zapatos para la acción creados por Edward de Bono. Los seis sombreros para pensar es un modelo de gran alcance y enfoque creativo para interactuar, y está destinado a mejorar los resultados de la reflexión y el debate. Está diseñado para ayudar a los individuos a explorar deliberadamente una variedad de perspectivas sobre un tema.

Los seis zapatos tiene como propósito establecer el estilo de la acción por adelantado, donde una persona pueda comportarse de una manera determinada ante un problema y así solucionarlo de una forma práctica, novedosa y útil.

El instrumento elaborado abarca tres dimensiones de análisis del grupo en el EVA (la cognitiva, la social y la mediadora), compuestas cada una de ellas por categorías y éstas, a su vez, por indicadores que usa el profesor para evaluar el contenido de la interacción y la actuación del estudiante en el foro del EVA.

- La cognitiva. Abarca lo relacionado con el contenido de la interacción de los estudiantes para adquirir, procesar, fijar, recuperar y utilizar la información recogida producto de las interacciones en el EVA y ponerlas en función de los procesos de aprendizaje y de formación y desarrollo del grupo.

- La social. Comprende lo referente a las relaciones afectivas, la orientación y el control con el grupo en el EVA.

- La mediadora. Incluye lo relativo a la presencia, guía, orientación y el control que realiza el profesor con el grupo y el empleo de las TIC para acceder a los recursos suministrados por el profesor en el EVA.

El profesor es un agente cultural y un mediador entre los productos socio-históricos y los procesos de apropiación de los estudiantes ${ }^{20}$. Es quien estructura las situaciones de aprendizaje y modos de actuar, plantea los retos, sugiere alternativas, ayuda, guía y evalúa en todo el proceso.

Para determinar las categorías e indicadores del instrumento diseñado se partió del estudio de los planteamientos de los profesores sobre ¿qué? y ¿cómo? debe de evaluarse en los foros. Fueron consultados 14 trabajos relacionados con el análisis de las interacciones, entre ellos: 
Lipponen, L. ${ }^{21}$; Gros, B. ${ }^{22}$; Domínguez, D. y Alonso, L. $^{23}$; Marcelo, C. y Perera, H. ${ }^{24}$; Cornejo, M. J. ${ }^{25}$; Ibarra, S. M. y Rodríguez, G. ${ }^{26}$; De Unigarro, M. A. ${\text { y } \operatorname{otros}^{27} \text {; Ribes, E. y otros }}^{28}$; Rebollo, M. y otros ${ }^{29}$ y como resultado se determinaron 14 categorías y 38 indicadores.

Otros criterios considerados para la determinación de las categorías e indicadores del instrumento fueron los obtenidos mediante la aplicación de la técnica de Grupo Focal con los estudiantes de quinto año de la carrera de Ingeniería en Infor- mática de la Universidad de Matanzas, Cuba. A partir de la aplicación de diferentes métodos se obtuvieron 12 indicadores.

Para ganar en la fiabilidad y validez de las categorías e indicadores utilizados en el instrumento confeccionado (Tabla N. ${ }^{\circ} 1$ ), se realizó un proceso de triangulación con la información recogida lo que ayudó a perfilar el resultado a partir de la coincidencia con la información obtenida de la revisión bibliográfica.

Tabla $\mathbf{N}^{\circ}$ 1. Instrumento los diez emoticones

\begin{tabular}{|c|c|c|c|c|}
\hline Dimensión & Categoría & Indicadores & Valor & Color \\
\hline \multirow{4}{*}{ Cognitiva } & Exploración & $\begin{array}{l}\text { - Hace preguntas con el objetivo de entender el } \\
\text { contenido objeto de aprendizaje. }\end{array}$ & 1 & Morado \\
\hline & Integración & $\begin{array}{l}\text { - Responde o indaga para clarificar, asocia ideas, } \\
\text { ilustra con referencias. }\end{array}$ & 2 & Amarillo \\
\hline & Razonamiento & $\begin{array}{l}\text { - Analiza las aportaciones hechas, interpreta, } \\
\text { justifica, fundamenta. }\end{array}$ & 3 & Carmelita \\
\hline & Resolución & $\begin{array}{l}\text { - Da respuesta a un planteamiento de la temática } \\
\text { objeto de discusión. }\end{array}$ & 4 & Verde \\
\hline \multirow{5}{*}{ Social } & \multirow{3}{*}{ Afectiva } & - Neutro, no participa en el foro. & 0 & Blanco \\
\hline & & $\begin{array}{l}\text { - Negativo, comportamiento en que se ataca } \\
\text { a otro miembro o se fortalece la posición } \\
\text { defensiva; bloquea o dificulta cada propuesta } \\
\text { o idea sin proponer una alternativa o sin } \\
\text { manifestar un desacuerdo argumentado. }\end{array}$ & 5 & Negro \\
\hline & & $\begin{array}{l}\text { - Expresiones de preferencias, emociones, } \\
\text { sensaciones, ánimo, agradecimiento, confianza, } \\
\text { apoyo, sostén y motivación en relación con el } \\
\text { aprendizaje. }\end{array}$ & 6 & Rojo \\
\hline & \multirow[b]{2}{*}{$\begin{array}{l}\text { Cohesión } \\
\text { grupal }\end{array}$} & - Exige disciplina y el cumplimiento de ella. & 7 & Rosado \\
\hline & & $\begin{array}{l}\text { - Resume o dirige los principales puntos del } \\
\text { debate, elabora conclusiones, hace declaración } \\
\text { consciente y directa de apoyo o de conformidad } \\
\text { con las ideas de los demás, aconseja, guía, } \\
\text { conduce. }\end{array}$ & 8 & Azul \\
\hline \multirow{2}{*}{$\begin{array}{l}\text { Mediadora } \\
\text { (Presencia } \\
\text { docente) }\end{array}$} & \multirow[t]{2}{*}{$\begin{array}{l}\text { Promueve, anima } \\
\text { la interacción }\end{array}$} & $\begin{array}{l}\text { - Convoca a que participen. Impulsa, estimula y } \\
\text { fomenta la ayuda mutua, regula las interacciones. }\end{array}$ & \multirow[t]{2}{*}{9} & \multirow[t]{2}{*}{ Arco Iris } \\
\hline & & - Respuesta al debate. & & \\
\hline
\end{tabular}

21 Lipponen, L. (2003).

22 Gros, B. (2004).

23 Domínguez, D. y Alonso, L. (2004).

24 Marcelo, C. y Perera, H. (2006).

25 Cornejo, M. J. (2006), pp. 277-297.

26 Ibarra, S. M. y Rodríguez, G. (2007).

27 De Unigarro, M. A. y otros (2007).

28 Ribes, E. y otros (2008).

29 Rebollo, M. y otros. (2008). 
En dependencia del valor de la evaluación que emite el profesor al estudiante, se le asigna el emoticón de un color determinado que lo representa en el diagrama estructural para su análisis. Dicha asignación se realiza con la herramienta informática, elaborada por los autores del trabajo, denominada Net@nalisis. El diagrama estructural es una representación gráfica de la posición de los actores de una red de interacción social ${ }^{30}$.

En el diagrama estructural los colores de los emoticones representan:

- Color morado: Representa al estudiante que interviene en el foro para buscar, reconocer, registrar.

- Color amarillo: Para el que compone, unifica.

- Color carmelita: Al que fundamenta, argumenta.

- Color verde: Al que da respuesta a un planteamiento de la temática objeto de discusión.

- Color blanco: Al estudiante observador, que aunque forma parte del grupo en el EVA no participa del debate en el foro.

- Color negro: Al negativo que evidencia pesimismo frente a las actividades a llevar a cabo.

- Color rojo: Cubre la intuición, las emociones y sensaciones.

- Color rosado: Para el estudiante que vela por el cumplimiento de las normas fijadas en el grupo, por la disciplina.

- Color azul: Alumno que conduce el proceso y aconseja.

Esta representación se obtiene después de que el profesor realizó el análisis del contenido de las interacciones y evalúa al estudiante en dependencia de su aporte o su modo de actuar. La evaluación se realiza en la medida que los estudiantes participan en el foro.

El profesor siempre lleva el emoticón color arco iris, que representa la consideración y reconocimiento de los demás, cuida de la capacidad de socialización del conocimiento y del aprendizaje de todos los miembros del grupo, respeta la independencia de los estudiantes, ayuda, orienta, controla y evalúa.

30 Troyano, F. y otros. (2005).

\section{Herramienta informática NET@NALISIS}

La herramienta Net@nalisis elaborada por los autores de este trabajo ofrece la representación gráfica del modelo de interacción del estudiante o del grupo que desarrolla las actividades en el foro del EVA. La misma permite obtener indicadores individuales y grupales según las medidas del análisis de redes sociales (ARS).

El modelo de interacción del estudiante es una representación a través de un diagrama estructural que recoge informaciones de quién y con quiénes se relaciona el estudiante para desarrollar las actividades, qué tipo de contribución está aportando al grupo según las dimensiones a evaluar en el foro (ver Tabla $\mathrm{N}^{\circ} 1$ ) para formar y desarrollar al grupo en el EVA. El modelo de interacción del grupo se obtiene a partir de la información contenida en los modelos de interacción individual.

Los diagramas estructurales son modelados como una red o grafo. Estas redes están compuestas por nodos que representan a los participantes en el foro, y por aristas que informan de las relaciones existentes entre ellos. Estos diagramas son de gran utilidad para conocer cómo se comporta la dinámica grupal. En el Gráfico $\mathrm{N}^{\circ} 1$ se puede apreciar parte de interfaz y funcionalidad de la misma.

Gráfico N. ${ }^{\circ}$ 1. Interfaz de la herramienta informática Net@nalisis.

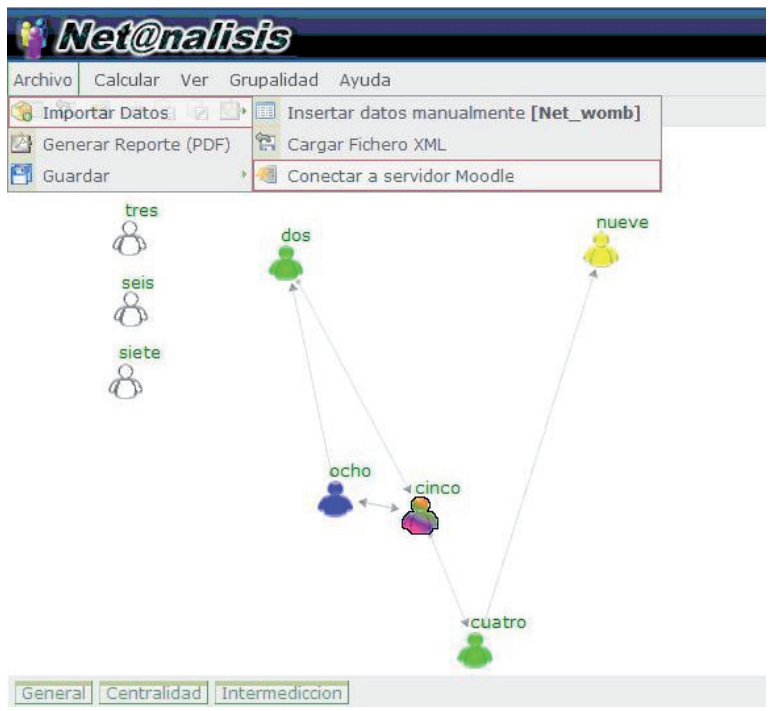

La herramienta informática está compuesta por varios paquetes:

- Net_womb: Plantilla que permite capturar los datos relacionales en forma de matriz de adyacencia, así como importar datos previamente 
guardados por Net@nalisis en formato XML, o datos exportados por otro software del ARS como por ejemplo UCINET.

- Net_@: Este paquete permite calcular indicadores del ARS.

- NetDraWeb: Programa gráfico destinado a la visualización del modelo de interacción del estudiante y del grupo en forma de grafo.

- Net_@_report: Programa que permite la exportación de las configuraciones de las interacciones que se establecen entre los estudiantes en el foro del EVA. El fichero creado contiene una imagen que representa el diagrama estructural del modelo de interacción del estudiante y del grupo, el resultado de los indicadores de ARS en forma tabular, y la muestra de las transformaciones matriciales de la red, así como el análisis estadístico en forma de gráficas de barras.

El proceso de validación de la herramienta informática Net@nalisis se realizó a través de la modelación de pruebas paralelas con el software profesional UCINET versión 6.0, un programa integrado que permite analizar matrices y visualizarlas.

El diagrama estructural del Gráfico N. ${ }^{\circ} 1$ muestra un ejemplo del modelo de interacción del grupo una vez aplicada el instrumento los diez emoticones, y el profesor realiza el monitoreo al mismo con la herramienta informática Net@nalisis. En este caso se puede apreciar a tres estudiantes (tres, seis y siete) representados por el emoticón color blanco, por lo que son considerados como neutros, que no participan del desarrollo de las actividades en el foro; dos estudiantes (dos y cuatro) con el emoticón color verde, por lo que sus intervenciones en el foro son evaluadas por el profesor según el instrumento los diez emoticones con el valor 4 dentro de la dimensión cognitiva, categoría resolución, porque da respuesta a un planteamiento de la temática objeto de discusión de forma correcta.

También apreciamos un estudiante (ocho) con el emoticón azul, lo cual quiere decir que fue evaluado por el profesor con el valor 8, aplicando el instrumento los diez emoticones porque su contribución en el foro es clasificada en la dimensión social, categoría cohesión grupal, en el indicador resume o dirige, los principales puntos del debate y elabora conclusiones.
Un estudiante es representado con el emoticón amarillo (nueve), significando que es clasificado en la dimensión cognitiva, en la categoría integración, porque responde o pregunta para clarificar, asocia ideas, ilustrar. El emoticón color arco iris representa al profesor (cinco), que actúa como mediador, facilitador del proceso de aprendizaje y de formación y desarrollo del grupo en el EVA.

También se puede observar en el diagrama estructural del Gráfico N. ${ }^{\circ} 1$ cómo son las interacciones en el grupo: en este caso el participante ocho interactúa con el dos y cinco, el dos con cinco, el cinco con ocho y cuatro, y el cuatro con nueve. Se puede apreciar que las interacciones pueden ser recíprocas o en un solo sentido.

\section{CONCLUSIONES}

1. El valor fundamental de los diez emoticones radica en su utilidad para detectar roles dentro del grupo en el desarrollo de las actividades en el foro del EVA, lo cual resulta útil para evitar las divagaciones y conflictos innecesarios.

2. Es un instrumento para el análisis del contenido de las interacciones y la actuación, que puede ser utilizado para facilitar la resolución y análisis de problemas desde distintos puntos de vista o perspectivas. Brinda un marco de referencia para el grupo que puede promover reflexiones inmediatas.

3. Entre los beneficios que aporta el instrumento los diez emoticones para el proceso de aprendizaje y la formación y desarrollo del grupo en el EVA se destacan un conjunto de acciones que puede promover el profesor, entre ellas:

- Fomentar las interacciones recíprocas.

- Promover las interacciones y la comunicación en toda su amplitud.

- Apoyar el proceso de aprendizaje.

- Definir actuaciones adecuadas y detectar actitudes negativas y resistencia.

- Despertar la motivación.

- Mejorar la actitud, la motivación y la satisfacción de los estudiantes en el desarrollo de actividades en la plataforma.

- Identificar y sortear los roles, las posiciones de los estudiantes en el grupo.

- Conocer como se manifiestan algunos de los elementos que caracterizan el trabajo en grupo en los EVA, entre ellos tenemos:

- La comunicación es rápida, concreta y clara. 
- La exposición de las ideas es clara, precisa y bien argumentada.

- Se trabaja en la concepción de un objetivo común.

- La interacción es estable en el tiempo.

- Se desarrollan lazos afectivos entre los estudiantes.

4. Algunos de los efectos que produce el instrumento en los estudiantes son:

- Los ayuda a reflexionar sobre su aprendizaje.

- Mejora su actuación en el desarrollo de actividades de aprendizaje.

- Fomenta la socialización del conocimiento.

- Posibilita organizar y conectar unos aprendizajes con otros.

- Desarrolla habilidades en la comunicación virtual.

5. La herramienta informática Net@nalisis resulta muy importante para el profesor a la hora de valorar los procesos de aprendizaje, formación y desarrollo del grupo en el EVA. Su contribución práctica es fundamental:

- Para analizar los modelos de interacción del estudiante y del grupo, lo que permite detectar los roles y posiciones de los integrantes en el EVA.

- Con la representación gráfica se visualizan las interacciones de los estudiantes en el EVA atendiendo a cuatro elementos de representación fundamentales para contribuir a la formación y desarrollo del grupo:

- La presencia, la participación del estudiante en una actividad.

- La identidad o filiación de los estudiantes, expresada por la socialización, por la compatibilidad de intereses, deseos mutuos por lograr los objetivos y aceptar las normas.

- La interacción- comunicación entre los estudiantes.

- La cohesión del grupo.

- Brinda la posibilidad de registrar y guardar la historia de las interacciones que ocurren para reflexionar sobre ellas, evaluarlas y mejorarlas.
- Posibilita al profesor observar cómo transcurre la dinámica grupal e interpretar los fenómenos observados que considere significativos, los que pueden discutirse en el grupo para producir un cambio en la dinámica a favor de los objetivos propuestos.

6. Para el estudiante, Net@nalisis es un medio que puede estimular a la reflexión sobre su propio comportamiento en el desarrollo de las actividades grupales, ya que posibilita conocer determinados comportamientos a partir de los modelos de interacción individual de sus compañeros, lo que puede ser válido para cambiar actitudes negativas, motivarse y, en algunos casos, para seleccionar al compañero adecuado cuando requiera asistencia.

\section{BIBLIOGRAFÍA}

\section{Internet}

AGUILAR, A. R. (2005). “Agentes Pedagógicos Virtuales Inteligentes. Una Estrategia para Entrenamiento de Equipos". En línea. Disponible en: http://is.ls.fi.upm.es/doctorado/ Trabajos20032004/Aguilar.pdf. Consultado en junio del 2008.

CORNEJO, M. J. (2006). "El análisis de las interacciones grupales: las aplicaciones Socios". En: Anuario de Psicología. Año 37, N. ${ }^{\circ} 3$. En línea. Disponible en: http://www.ub.edu/ dppss/lps/docu/tag.pdf. Consultado el 27 de enero del 2007.

COSTAGUTA, R. (2006). “Una Revisión de Desarrollos Inteligentes para Aprendizaje Colaborativo Soportado por Computadora". Argentina. En: Revista Ingeniería Informática . N. ${ }^{\circ}$ 13. En línea. Disponible en: http://www. inf.udec.cl/revista. Consultado el 14 de diciembre del 2007.

DE UNIGARRO, M. A. y otros. (2007). "Conformación de una comunidad virtual de aprendizaje, a partir de un proceso de formación de maestros universitarios". En: RED: Revista de Educación a Distancia. Año 18, N. ${ }^{\circ} 1$. En línea. Disponible en: http://www.um.es/ead/ red/18/prado.pdf. Consultado el 11 de junio del 2008.

DOMÍNGUEZ, D. Y ALONSO, L. (2004). "Metodología para el análisis didáctico de foros virtuales". En línea. Disponible en: http:// 
edutec2004.Imi.ub.es/pdf/46.pdf. Consultado el 20 de diciembre del 2005.

EHULETCHE, M. A. y otros (2007). "Ajustes pedagógicos en entornos virtuales: los procesos colaborativos". En línea. Disponible en: http:// www.utn.edu.ar/aprobedutec07/docs/148.doc. Consultado en enero del 2009.

ERIC, B. (2008). "Teacher development, discussion lists and forums: issues and results". En línea. Disponible en: http://www.editlib. org/p/27675. Consultado el 20 de diciembre del 2008.

FUENTES, M. (2001). “¿Por qué el grupo en la psicología social?” Disponible en: http://www. dict.uh.cu/Revistas/PS2000_2001/. Consultado en mayo del 2007.

GARCÍA, M. C. (2006). "Pregunta cuando quieras. La interacción didáctica en los nuevos ambientes virtuales de aprendizaje". En línea. Disponible en: http://www.elearningeuropa. info/directory/index.php. Consultado en junio del 2008.

GROS, B. Y SILVA, J. (2007). "The problem of analysing asynchronous discussions in computer supported collaborative learning". En línea. Disponible en: http://www. um.es/ead/red/16/gros.pdf. Consultado el 18 de junio del 2008.

IBARRA, S. M. Y RODRÍGUEZ, G. (2007). "El trabajo colaborativo en las aulas universitarias: reflexiones desde la autoevaluación". En línea. Disponible en: http://www.revistaeducacion.mec.es/re344_15.html. Consultado el 18 de julio del 2008.

LUGO, L. (2009). "Análisis de las interacciones en los Foros de Moodle con software libre, específicamente con Agna, Moodle Forum SNA y yED”. En línea. Disponible en: http:// edusol.info/es/bitacora/larrylugo/herramientaspara-analisis-redes-sociales. Consultado el 24 de noviembre del 2009.

MARCELO, C. Y PERERA, H. V. (2006). "Didactic interaction in e-learning: New styles for new environments". En línea. Disponible en: prometeo.us.es/idea/index.php?option $=$ com_conte $n t \&$ task $=$ view\&id $=201$ \&ltemid . Consultado el 14 de julio del 2008.

MARTÍNEZ, A. (2003). "Método y modelo para el apoyo computacional a la evaluación en
CSCL". Tesis de Doctorado. Departamento de Informática Universidad de Valladolid. En línea. Disponible en: www.cibernetia. com/tesis_es/. Consultado el 11 de marzo del 2005.

MONGUET, J. (2008). "Monitorización del progreso en el aprendizaje". Tesis de Doctorado. Universitat Politécnica de Catalunya. Barcelona. En línea. Disponible en: www.tesisenxarxa.net/TESIS_UPC/...//01. Consultado en julio del 2008.

PAZ, V. (2007). “Looking for evidence of learning: Assessment and analysis methods for online discourse". En: Computers in Human Behavior. Año 24, N. ${ }^{\circ}$ 2. En línea. Disponible en: http://www.sciencedirect.com/science?

REBOLLO, M. y otros. (2008). "Las emociones en el aprendizaje online”. En: Revista Relieve. Año 14, N. ${ }^{\circ} 1$. En línea. Disponible en: http:// www.uv.es/RELIEVE/v14n1/. Consultado en enero del 2009.

RIBES, E. y otros (2008). "Análisis teórico de las dimensiones funcionales del comportamiento social”. En línea. Disponible en: http://lodel. irevues.inist.fr/cahierspsychologiepolitique/ index.php?id $=807$. Consultado el $20 \mathrm{de}$ enero del 2009.

RODRÍGUEZ, M. (2008). "Una estrategia para el diseño e implementación de cursos virtuales de apoyo a la enseñanza semipresencial en la carrera de Economía de la Universidad de Camaguiey". Tesis de Doctorado. Tesis presentada en opción al grado científico de Doctor en Ciencias de la Educación.

SILVA, J. (2007). "Intervención en un entorno virtual de aprendizaje para la formación continua de docentes de la enseñanza básica”. Tesis de Doctorado. Disponible en: $w w w$. tesisenxarxa.net/TESIS_UB/AVAILABLE/TDX0713107-120211//01.JESQ_Cap\%EDtulo_I.pdf. Consultado el 12 de diciembre del 2008.

SOLLER, A. y otros (2005). "From Mirroring to Guiding: A Review of State of the Art Technology for Supporting Collaborative Learning". En: International Journal of Artificial Intelligence in Education. N. ${ }^{\circ}$ 15. En línea. Disponible en: http://portal.acm.org/citation.cfm?id=1 434935.1434937\& coll = GUIDE\&dl = GUIDE $\delta C F I D=57655873 \& C F T O K E N=91105323$. Consultado el 12 de abril del 2008. 
TROYANO, F. y otros (2005). "Análisis de Redes Social mediante Diagramas Estratégicos y Diagramas Estructúrale". En: REDES- Revista hispana para el análisis de redes sociales. Año 8, N. ${ }^{\circ}$ 2. En línea. Disponible en http:// revista-redes.rediris.es. Consultado el 21 de noviembre del 2007.

VÉLEZ, O. y otros (2004). "Una revisión del estado de los sistemas Cooperativos Multiagente en la tutoría y la formación”. En línea. Dis- ponible en: http://lsm.dei.uc.pt/ribie/docfiles/ txt2003729192151paper-064.pdf. Consultado el 18 de julio del 2008.

YOO, J., y otros (2009). "Improving Student Performance by Enforcing Algorithm Development". En: Proceedings of World Conference on Educational Multimedia, Hypermedia and Telecommunications. En línea. Disponible en: http://www.editlib.org/p/31962. Consultado el 20 de septiembre del 2009. 